\title{
Clinical cross-reactivity to quail's egg in patients with hen's egg allergy
}

Masatoshi Mitomori ${ }^{1}$, Noriyuki Yanagida ${ }^{1}$, Mari Takei $^{1}$, Kinji Tada ${ }^{1}$, Makoto Nishino ${ }^{1}$, Sakura Sato ${ }^{1}$, and Motohiro Ebisawa ${ }^{1}$

${ }^{1}$ National Hospital Organization, Sagamihara National Hospital

September 25, 2021

\section{Clinical cross-reactivity to quail's egg in patients with hen's egg allergy}

Masatoshi Mitomori ${ }^{\mathrm{a}}$, Noriyuki Yanagida ${ }^{\mathrm{a}}$, Mari Takei ${ }^{\mathrm{b}}$, Kinji Tada ${ }^{\mathrm{b}}$, Makoto Nishino $^{\mathrm{a}}{ }^{\mathrm{c}}$, Sakura Sato $^{\mathrm{b}}$, Motohiro Ebisawa ${ }^{\mathrm{b}}$

${ }^{a}$ Department of Pediatrics, National Hospital Organization, Sagamihara National Hospital, Kanagawa, Japan

${ }^{\mathrm{b}}$ Clinical Research Center for Allergy and Rheumatology, National Hospital Organization, Sagamihara National Hospital, Kanagawa, Japan

${ }^{c}$ Course of Allergy and Clinical Immunology, Juntendo University Graduate School of Medicine, Tokyo, Japan

Running title: Clinical cross-reactivity of HE \& QE

Corresponding author:

Noriyuki Yanagida

18-1, Sakuradai, Minamiku, Sagamihara, Kanagawa 252-0392, Japan

Tel.: +810427428311 Fax: +810427425314

E-mail: yana@foodallergy.jp

Word count:1153

No. of Tables: 1

No. of Figures: 2

Material in electronic repository: 1 table and 1 figure

i. A statement with potential conflict of interests related to the manuscript content:

Motohiro Ebisawa received lecture fees from DBV Technologies and Mylan EPD. Sato Sakura received lecture fees from Mylan EPD. The other authors declare that they have no conflicts of interest.

ii. Financial support: None.

iii. Keywords

hen's egg, quail's egg, food allergy, cross-reactivity, oral food challenge, child, pediatric

iv. Main text: 
To the Editor,

Hen's egg (HE) is consumed worldwide and is one of the most frequent causes of food allergy. ${ }^{1,2}$ In Japan, $\mathrm{HE}$ is consumed most frequently among avian eggs, followed by quail's egg (QE). Quails (Coturnix japonica ) belong to the same Galliformes Phasianidae family as chicken (Gallus gallus domesticus ); therefore, crossreactivity may occur between $\mathrm{HE}$ and QE. Consequently, $\mathrm{HE}$ allergic patients are generally advised to avoid $\mathrm{QE}$ consumption. However, there has been only one report on the serological cross-reactivity of $\mathrm{HE}$ and $\mathrm{QE}^{3}$ and one case report on clinical cross-reactivity. ${ }^{4}$ Moreover, whether all HE allergic patients need to avoid QE consumption remains unclear. This study, therefore, aimed to evaluate the clinical cross-reactivity between $\mathrm{HE}$ and QE by performing oral food challenge (OFC) tests.

We conducted a prospective study to determine whether HE allergic patients show cross-reactivity to QE. We performed QE-OFC in patients with HE allergy between January 2018 and October 2019. HE allergy was diagnosed through OFCs, which were performed stepwise, starting from a low-dose HE-OFC (containing $1 / 25$ of a heated $\mathrm{HE}$ ). Patients with negative results proceeded to a medium-dose HE-OFC (containing $1 / 8$ of a heated HE). Patients with positive low-dose or medium-dose HE-OFC were included in this study. Patients with missing clinical data were excluded. QE-OFC was performed using one heated QE. All OFCs were performed in a single-dose or two-dose administration. In the two-dose OFCs, the challenged food was distributed at 60-min intervals. Symptom severity during OFCs was assessed according to the Japanese guidelines for food allergy. ${ }^{2}$ Serum levels of total immunoglobulin E (IgE) and specific IgE to HE white (HEw-sIgE), HE ovomucoid (OVM-sIgE), and QE white (QEw-sIgE) (ImmunoCAP ${ }^{\mathrm{TM}}$; Thermo Fisher Scientific/Phadia, Uppsala, Sweden) were measured within four months of conducting QE-OFC, and skin prick test (SPT) was performed during QE-OFC. The SPT procedure is described in Supplemental Methods. This study was approved by the ethics committee of Sagamihara National Hospital (approval number: 2017-021), and was registered at the University Hospital Medical Information Network Clinical Trials Registry (no: UMIN000034820). The written informed consent was obtained from the guardians of all patients.

Among 870 patients who underwent HE-OFC, 183 failed in the low-dose or medium-dose HE-OFC, 22 of whom underwent a QE-OFC. Two patients were excluded because of missing clinical data, and the remaining 20 were enrolled (Figure 1). Median patient age was 2.9 (range, 1.0-16.4) years, and nine (45\%) patients had a history of anaphylaxis to HE. The median HEw-sIgE, OVM-sIgE, and QEw-sIgE levels were 9.95 $(2.67-365), 8.66(<0.10-191)$, and $4.15(0.73->100) \mathrm{kU}_{\mathrm{A}} / \mathrm{L}$, respectively (Table 1$)$. There was a correlation between HEw-sIgE and QEw-sIgE levels (Supplemental Figure 1). SPT was performed in 13 patients, and $12(92 \%)$ were found to be sensitized to QE. The median SPT wheal diameters for HEw and QEw were 15 $(3-32)$ and $12(0-25) \mathrm{mm}$, respectively.

Among the 20 patients, four reacted to the low-dose HE-OFC (threshold dose, $1 / 25$ of a heated HE or lower), and 16 passed the low-dose HE-OFC but reacted to the medium-dose HE-OFC (threshold dose, $>1 / 25-1 / 8$ of a heated HE). Of the 20 patients, 9 (45\%) failed in the QE-OFC. The median interval between QE-OFC and HE-OFC was 2.8 months. The rate of positive QE-OFC results was significantly higher in patients with a threshold dose of [?]1/25 of a heated HE than in those with a threshold dose of $>1 / 25-1 / 8$ of a heated HE $(100 \%[4 / 4]$ vs. $31 \%$ [5/16], $p=0.026$; Figure 2). Patients' symptoms, severity, and treatment during the QE-OFC are described in Supplemental Table 1. Three patients presented with symptoms in multiple organs; however, their symptoms improved after treatment with antihistamine and steroid or inhalation of $\beta_{2}$ stimulants. The HEw-sIgE, OVM-sIgE, and QEw-sIgE levels were significantly higher in patients with a positive QE-OFC result than in those with a negative QE-OFC result. There was no significant difference in the SPT wheal diameter to HEw and QEw between patients who reacted to QE-OFC and those who did not (Table 1).

To our knowledge, this is the first prospective study to evaluate the clinical cross-reactivity between HE and QE through OFC tests. In this study, clinical cross-reactivity to QE was observed in almost half of the patients who were allergic to HE.

In a previous case report, a patient with HE allergy showed cross-reactivity to QE. ${ }^{4}$ Furthermore, approxi- 
mately $70 \%$ of HE allergic children had a positive SPT result to $\mathrm{QEw}^{5}$; however, whether they showed clinical cross-reactivity to QE was not confirmed. In our study, $92 \%$ of the HE allergic patients had a positive SPT result to $\mathrm{QEw}$, and $45 \%$ reacted to one $\mathrm{QE}$.

The mean weight of HE and QE is $58 \mathrm{~g}$ and $11.3 \mathrm{~g}$, respectively. ${ }^{6}$ Because the ratio of egg white and egg yolk mass in $\mathrm{HE}$ is almost equivalent to that of $\mathrm{QE}^{6,7}$ and the percentage of protein in $\mathrm{HEw}$ is also equivalent to that of $\mathrm{QEw},{ }^{6}$ the amount of protein in the egg white of one QE would be equivalent to approximately $1 / 5$ of that in one HE. Since the main antigen of immediate-type egg allergy is egg white, if the antigenicity of $\mathrm{HEw}$ and QEw is deemed to be identical, all $\mathrm{HE}$ allergic patients with a threshold dose of [?]1/8 of a heated HE would be expected to fail a QE-OFC. However, in this study, while all the patients with a threshold dose of [?]1/25 of a heated HE failed the QE-OFC, only $31 \%$ patients with a threshold dose of $>1 / 25-1 / 8$ of a heated HE failed. Therefore, QEw antigenicity seemed to be lower than that of HEw when comparing in terms of the same amount of protein, and whether $\mathrm{HE}$ allergic patients reacted to one $\mathrm{QE}$ appeared to depend on their threshold of HE.

This study has some limitations. First, OFCs were not double-blind and placebo-controlled. However, as most patients exhibited objective symptoms during OFCs, the number of false-positive OFC results would be low. Second, some patients may have achieved tolerance during the interval between the OFCs. However, the interval between QE-OFC and HE-OFC was relatively short, with a median of 2.8 months. Moreover, the median age of the subjects in this study was 2.9 years, and since it has been reported that only $30 \%$ of Japanese HE allergic patients achieve tolerance between 3 and 6 years old, ${ }^{8}$ the influence of the interval between the OFCs is considered minimal. Third, we did not evaluate whether patients with negative QEOFC results were reactive to amounts larger than one QE. Therefore, our study may have underestimated the rate of patients who had true clinical cross-reactivity to QE. Further studies with a larger number of patients, including those with a higher HE threshold, are necessary.

In conclusion, this study showed that some HE allergic patients are clinically cross-reactive to QE. Furthermore, patients with a lower threshold of HE were more likely to react to QE. Therefore, HE allergic patients, especially those with a low threshold, should consider avoiding QE consumption.

Masatoshi Mitomori, MD ${ }^{\mathrm{a}}$, Noriyuki Yanagida, MD ${ }^{\mathrm{a}}$, Mari Takei, MD ${ }^{\mathrm{b}}$, Kinji Tada, MD ${ }^{\mathrm{b}}$, Makoto Nishino, $\mathrm{MD}^{\mathrm{a}, \mathrm{c}}$, Sakura Sato, MD ${ }^{\mathrm{b}}$, Motohiro Ebisawa, MD, $\mathrm{PhD}^{\mathrm{b}}$

${ }^{a}$ Department of Pediatrics, National Hospital Organization, Sagamihara National Hospital, Kanagawa, Japan

${ }^{\mathrm{b}}$ Clinical Research Center for Allergy and Rheumatology, National Hospital Organization, Sagamihara National Hospital, Kanagawa, Japan

${ }^{\mathrm{c}}$ Course of Allergy and Clinical Immunology, Juntendo University Graduate School of Medicine, Tokyo, Japan.

\section{v. Acknowledgments}

We thank Editage (www.editage.jp) for their assistance with the English language editing.

\section{vi. Impact statement}

Some patients with allergy to hen's egg are also clinically reactive to quail's egg. Therefore, such patients, especially those with a low threshold dose, should consider avoiding the consumption of quail's egg.

\section{vii. References}

1. Sicherer SH, Sampson HA. Food allergy: A review and update on epidemiology, pathogenesis, diagnosis, prevention, and management. J Allergy Clin Immunol 2018;141:41-58.

2. Ebisawa M, Ito K, Fujisawa T. Committee for Japanese Pediatric Guideline for Food Allergy, The Japanese Society of Pediatric Allergy and Clinical Immunology, Japanese Society of Allergology, Japanese Guidelines for Food Allergy 2020. Allergol Int 2020;69;370-386. 
3. Takahashi K, Horiguchi M, Bando N, Tsuji H, Ogawa T, Asao T. Immunochemical characterization of ovomucoid from Japanese quail egg white using monoclonal antibodies. J Nutr Sci Vitaminol (Tokyo) 1999;45:491-500.

4. Alessandri C, Calvani M Jr, Rosengart L, Madella C. Anaphylaxis to quail egg. Allergy 2005;60:128129.

5. Moghtaderi M, Nabavizadeh SH, Hosseini Teshnizi S. The frequency of cross-reactivity with various avian eggs among children with hen's egg allergy using skin prick test results: fewer sensitizations with pigeon and goose egg. Allergol Immunopathol (Madr) 2020;48:265-269.

6. Tolik D, Poławska E, Charuta A, Nowaczewski S, Cooper R. Characteristics of egg parts, chemical composition and nutritive value of Japanese quail eggs-a review. Folia Biol (Krakow) 2014;62:287-292.

7. Standard Tables of Food Composition in Japan 2020 (Eighth Revised Edition)

8. Ohtani K, Sato S, Syukuya A, et al. Natural history of immediate-type hen's egg allergy in Japanese children. Allergol Int 2016;65:153-157.

\section{viii. Tables}

Table 1. Characteristics of the study patients and comparison of patients with QE-OFC positive and negative results

\begin{tabular}{lllll}
\hline Characteristics & $\begin{array}{l}\text { All patients } \\
\mathbf{n}=\mathbf{2 0}\end{array}$ & $\begin{array}{l}\text { QE-OFC positive } \\
\mathbf{n = 9}\end{array}$ & $\begin{array}{l}\text { QE-OFC negative } \\
\mathbf{n = 1 1}\end{array}$ & $p$ value* \\
Age (years) & $2.9(1.0-16.4)$ & $2.6(1.0-11.1)$ & $3.2(1.0-16.4)$ & 0.656 \\
Sex (male) & $14(70)$ & $7(78)$ & $7(64)$ & 0.642 \\
History of anaphylaxis to HE & $9(45)$ & $5(56)$ & $4(36)$ & 0.653 \\
Food allergy other than HE, current & $9(45)$ & $3(33)$ & $6(55)$ & 0.406 \\
Atopic dermatitis, current & $16(80)$ & $8(89)$ & $8(73)$ & 0.591 \\
Bronchial asthma, current & $3(15)$ & $1(11)$ & $2(18)$ & $>0.999$ \\
Allergic rhino-conjunctivitis, current & $3(15)$ & $0(0)$ & $3(27)$ & 0.218 \\
Total IgE $(\mathrm{kIU} / \mathrm{L})$ & $322(31.1-4040)$ & $526(58.4-1170)$ & $280(31.1-4040)$ & $>0.999$ \\
HEw-sIgE $(\mathrm{kU} / \mathrm{L})$ & $9.95(2.67-365)$ & $62.3(5.43-365)$ & $8.22(2.67-33.5)$ & 0.025 \\
OVM-sIgE $(\mathrm{kU} / \mathrm{L} / \mathrm{L})$ & $8.66(<0.10-191)$ & $53.2(3.22-191)$ & $4.57(<0.10-18.7)$ & 0.004 \\
QEw-sIgE $(\mathrm{kU} / \mathrm{A} / \mathrm{L})$ & $4.15(0.73->100)^{\mathbb{I}}$ & $36.8(2.75->100) \mathbb{I}$ & $3.28(0.73-14.2)^{+}$ & 0.019 \\
SPT wheal diameter to HEw $(\mathrm{mm})$ & $15(3-32)$ & $23(11-32)^{++}$ & $14(3-22)^{\S}$ & 0.101 \\
SPT wheal diameter to QEw $(\mathrm{mm})$ & $12(0-25)$ & $13(10-25)^{++}$ & $11(0-24)^{\S}$ & 0.295 \\
\hline
\end{tabular}

Values are reported as median (range) or number (\%).

* Comparisons between subjects with QE-OFC positive and negative results were conducted using MannWhitney $U$ test for continuous variables or using chi-square or Fisher's exact test for categorical variables.

${ }^{+}$In 2 patients, QEw sIgE data were missing

${ }^{++}$In 3 patients, SPT data were missing.

${ }^{\S}$ In 4 patients, SPT data were missing.

$\mathbb{I}_{\text {QEw-SIgE concentrations exceeding } 100 \mathrm{kU}} / \mathrm{L}$ were not evaluated by dilution.

Abbreviations: QE-OFC, quail's egg oral food challenge; HE, hen's egg; HEw, hen's egg white; OVM, hen's egg ovomucoid; QEw, quail's egg white; IgE, immunoglobulin E; sIgE, specific IgE; SPT, skin prick test

\section{ix. Figure legends}

Figure 1. Flowchart of the study participation selection process

Abbreviations: HE, hen's egg; QE, quail's egg; OFC, oral food challenge 
Figure 2. Positive rates in quail's egg oral food challenge.

Abbreviations: HE, hen's egg; QE, quail's egg; OFC, oral food challenge

\section{Author Contributions}

Masatoshi Mitomori: Conceptualization (equal); data curation (lead); formal analysis (lead); investigation (lead); methodology (lead); project administration (lead); resources (lead); software (equal); supervision (equal); validation (supporting); visualization (supporting); writing-original draft (lead); writing-review and editing (equal). Noriyuki Yanagida: Conceptualization (lead); data curation (supporting); formal analysis (supporting); investigation (equal); methodology (equal); project administration (equal); resources (equal); software (equal); supervision (lead); validation (lead); visualization (lead); writing-original draft (equal); writing -review and editing (lead). Mari Takei; Conceptualization (supporting); data curation (supporting); formal analysis (supporting); investigation (equal); methodology (supporting); project administration (supporting); resources (supporting); software (supporting); supervision (supporting); validation (supporting); visualization (supporting); writing-original draft (supporting); writing-review and editing (supporting). Kinji Tada: Conceptualization (supporting); data curation (supporting); formal analysis (supporting); investigation (equal); methodology (supporting); project administration (supporting); resources (supporting); software (supporting); supervision (supporting); validation (supporting); visualization (supporting); writing-original draft (supporting); writing-review and editing (supporting). Makoto Nishino: Conceptualization (supporting); data curation (supporting); formal analysis (supporting); investigation (equal); methodology (supporting); project administration (supporting); resources (supporting); software (supporting); supervision (supporting); validation (supporting); visualization (supporting); writing-original draft (lead); writing-review and editing (equal). Sakura Sato: Conceptualization (lead); data curation (supporting); formal analysis (supporting); investigation (equal); methodology (equal); project administration (equal); resources (equal); software (equal); supervision (lead); validation (lead); visualization (lead); writing-original draft (equal); writingreview and editing (lead). Motohiro Ebisawa: Conceptualization (lead); data curation (supporting); formal analysis (supporting); investigation (equal); methodology (equal); project administration (equal); resources (equal); software (equal); supervision (lead); validation (lead); visualization (equal); writing-original draft (equal); writing-review and editing (lead).

\section{Key Message}

This study showed that some hen's egg allergic patients are also clinically reactive to quail's egg. Therefore, such patients, especially those with a low threshold dose, should consider avoiding the consumption of quail's egg.

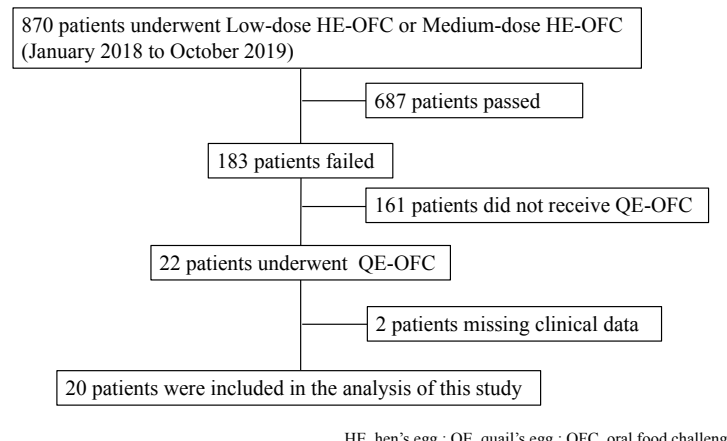




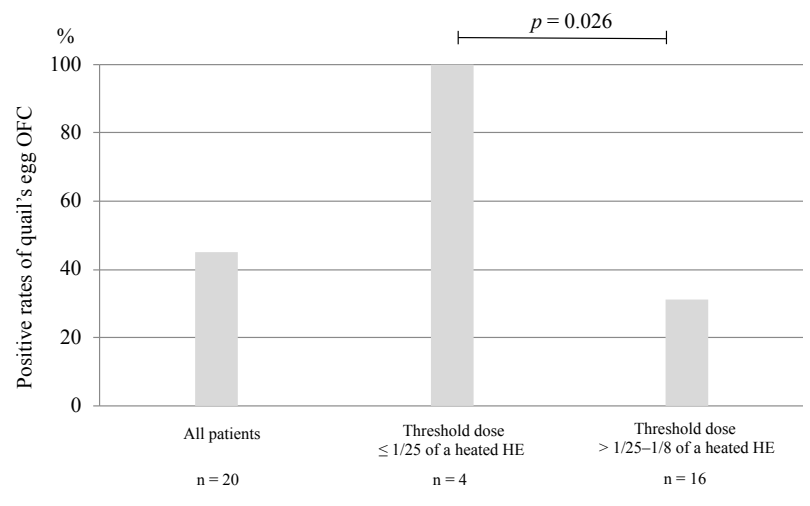

